\title{
A Minimum Toe-clearance Detector for Tripping Prediction
}

\author{
DAIKI HAMADA* ${ }^{*} \quad$ Member, $\quad$ Shota NAKAShima ${ }^{\dagger}$ Member \\ TOMOHIRo Morio $^{\dagger}$ Member, TATSuya Sugimura ${ }^{\dagger}$ Member \\ KAZUO HARUYAMA ${ }^{\ddagger *}$ Member
}

(Received November 21, 2019, revised January 8, 2020)

\begin{abstract}
Elderly people are at the risk of serious injuries by falling. As most of falls result from tripping, researchers assess the ease of tripping using the minimum toe clearance (MinTC) measure, which is known to be more variable in the elderly than in younger people. This paper proposes a method that judges the ease of tripping from the MinTC height measured by a One Dimensional Brightness Distribution Sensor (Obrid-Sensor). Using our method, we detected the difference between normal and dangerous gaits, and found significant differences between two gait types. In conclusion, our proposed system clearly distinguished normal from dangerous gaits. In future work, the method will be further adapted to actual environments by widening its detection area.
\end{abstract}

Keywords: Fall, Elderly, Gait, Obrid-Sensor, Minimum Toe Clearance,

\section{Introduction}

Fall prediction is a problem that needs immediate attention. Every year, approximately $20 \%$ of Japanese people aged over 65 fall more than once [1]. Experiencing falls instills resistance to daily life and reduces daily activities, including moving and changing clothes. As a result, many elderly people are bedridden and require nursing care. Nowadays, approximately 646,000 of elderly people die in one year because of falling accidents; indeed, falls are the second leading cause of unintentional injury leading to death, after road traffic injuries [2].

The factors of falls are classified into two categories: internal factors that relate to the physical ability of subjects and external factors that relate to the ambient environment. Regarding the internal factors, researchers have focused on medical conditions [3], history of falls [4], and dual tasks [5]. External factors, such as footwear [6] and visual information [7], cause fall-risky behaviors such as tripping or turning downward. Tripping while walking is a major cause of falls [8] and can often occur indoors [8]. Especially, many elderly people trip in indoor space such as living rooms and bedrooms [9], where they spend the majority of their time.

Current fall-prevention methods are scientifically tested evaluations of fall risk, such as the functional reach test, timed-up-go-test and standing-on-one-leg test, which are followed by physiotherapy-based treatments [10]. Other fall prevention methods use walking-correction apparatuses with light-feedback devices [11], and determine the risk of downturn from the bed by sensors placed around the bed

\footnotetext{
$\doteqdot$ Corresponding: haruyama@ube-k.ac.jp

* National Institute of Technology Ube College

2-14-1, Tokiwadai, Ube, Yamaguchi, Japan 755-8555

$\dagger$ Yamaguchi University

2-16-1, Tokiwadai, Ube, Yamaguchi, Japan 755-8611
}

[12].However, these methods are valid only for patients who try to prevent their own falls. They are implemented only when triggered by self-recognition of the user's fall risk. Therefore, walking data acquired in living rooms would be useful for assessing the most likely situations for tripping.

In this study, we collect walking data and analyze the tripping defined as the height between the toe and ground during walking. Walking data are usually collected using motion tracking systems [13], reaction force plates [14] and acceleration and inertial sensors [15]. However, motion tracking systems and reaction force plates are large and cumbersome, whereas acceleration sensors are troublesome to mount. Therefore, they are unsuitable for continuous data acquisition in daily life. Based on the Toe Clearance (TC), we instead propose a system that assesses the ease of tripping using an Obrid-Sensor that does not contact and restrain the user.

\section{Method}

2.1 Toe Clearance During walking, each leg cycles between two phases, the swing phase and the stance phase [16]. The swing phase begins at Toe-Off (TO) from the ground and ends at Heel-Strike (HS). Conversely, the stance phase begins at HS and terminates at TO. The stance phase is divided into three durations: Loading response (Load), Foot-Flat (FF), and Push-off (Push), which define the durations from HS to Toe-Strike (TS), from TS to Heel-Off (HO), and from HO to TO, respectively. Tripping is defined as touching the ground during the swing phase. Fig. 1 illustrates changes of left leg viewed from the right side. The durations of these phase were calculated as Eq. 1, Eq. 2, Eq. 3 and Eq. 4 [17]:

$$
\begin{aligned}
& \text { Load }=t(\mathrm{TS})-t(\mathrm{HS}) \\
& \mathrm{FF}=t(\mathrm{HO})-t(\mathrm{TS})
\end{aligned}
$$




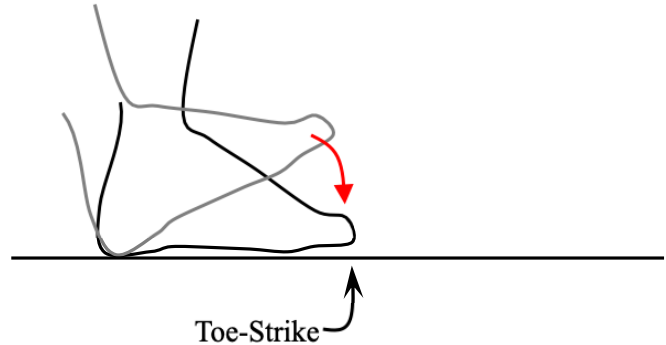

(a) Load

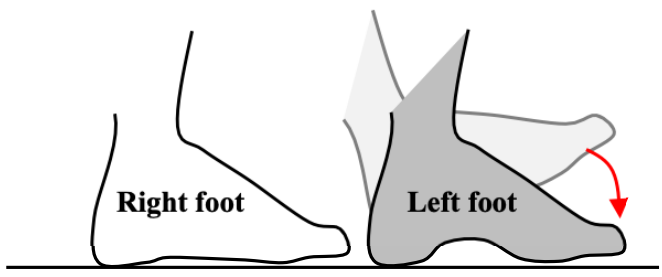

(b) Foot-Flat

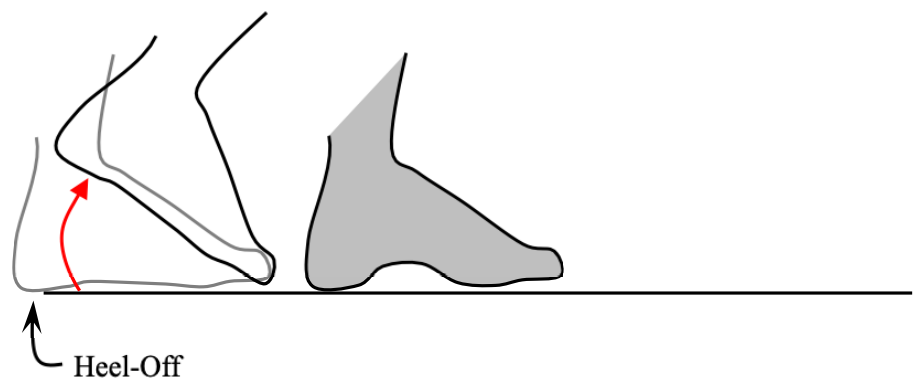

(c) Push

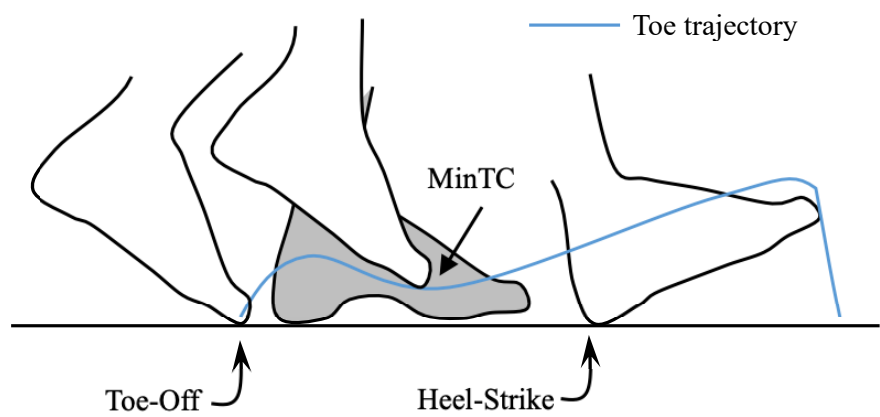

(d) Swing

Figure 1: Gait phases of human walking.

$$
\begin{aligned}
& \text { Push }=t(\mathrm{TO})-t(\mathrm{HO}) \\
& \text { Swing }=t(\mathrm{HS})-t(\mathrm{TO})
\end{aligned}
$$

where $t($.$) is the occurrence instant of the event.$

The Toe Clearance (TC) is the height between the toe and ground during walking. Between the heel and the ground is the Heel Clearance (HC). Fig. ?? shows the trajectories of TC and HC during the swing phase. The lowest TC, called the minimum TC (MinTC), is a recognized important index of easiness of tripping. According to Begg et al.[18], MinTC is seen in approximately half of the swing phases in healthy walkers. Applying the coordinate model based on the measured values, Winter [19] reported that the TC increases by $0.45 \mathrm{~cm}$ for each $1.4^{\circ}$ increment of the ankle angle.

2.2 Analysis of Tripping Mills et al.[20] reported no differences in the mean MinTCs of the young and elderly walkers; however statistical variability (assessed using the interquartile range) of the MinTC significant differed between the two groups [20]. Therefore, we considered that the TC has two patterns, one with high MinTC, the other with small MinTC. Recently, researchers have noted that when the MinTC is small, elderly people will likely fall by tripping when their toe touches the ground [21]. Therefore, by counting the frequency of small MinTC trajectories, we can estimate the ease of tripping. Many studies have reported mean MinTCs ranging from $10 \mathrm{~mm}$ to $24 \mathrm{~mm}$ [22]. Therefore, we set the MinTC threshold to the worst reported value (i.e., to $10 \mathrm{~mm}$ ). Below this threshold, the gait was judged as a small MinTC trajectory with a high fall risk.

2.3 One-Dimensional Brightness Distribution Sensor The data for the MinTC detection were collected by a One-
Dimensional brightness distribution Sensor (Obrid-Sensor). The Obrid-Sensor [23] is a human-shape detector with privacy preservation. The sensor comprises a cylindrical lens and a line sensor. The convex cylindrical lens depends on the light direction. Panels (a), (b), (c) of Fig. 2 are schematics of the overall view, side view, and top view of the sensor, respectively. From the side, the cylindrical lens looks like a flat panel that projects the vertical brightness irradiated from different positions onto the sensor (Fig. 2(b)). From the top, one observes the horizontal brightness irradiated from different positions and focused on the sensor (Fig. 2(c)). As shown in Fig. 2(a), the light radiated from Points $\mathrm{R}, R^{+}, R^{-}$on the subject's right side is focused on Point SR of the line sensor. Similarly, the light radiated from Points $C^{+}, \mathrm{C}$ and $C^{-}$(along the vertical line of symmetry of the subject) converges at Point $S_{C}$ of the line sensor, and that radiated from Points $L^{+}, \mathrm{L}$ and $L^{-}$on the subject's left side converges at Point $S_{L}$. Therefore, the output from the line sensor captures the horizontal distribution of the brightness from different vertical points.

To obtain the parameters from using the Obrid-Sensor data, we subtracted the background. Fig. 3 displays the correspondence between the subject and brightness distributions of the background, subject, and difference between the background and subject brightness. First, the sensor collects the brightness distribution of the empty room. Second, it collects the current brightness distribution with the subject present (i.e., the subject brightness distribution). The absolute difference between the two brightness distributions gives the characteristic parameter of sensor. The one-dimensional distribution reflects the position of the subject. The peak brightness depends on the color, shape, and 


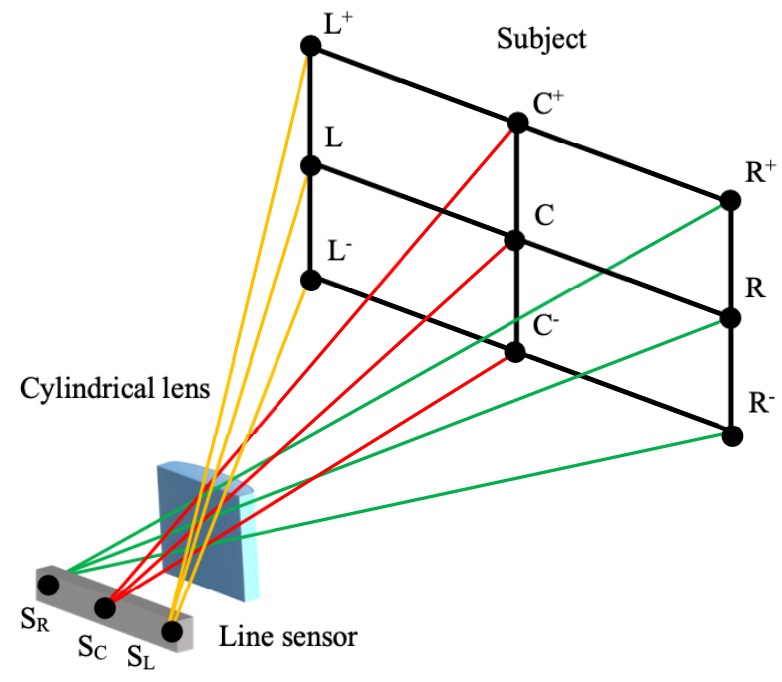

(a) Over view

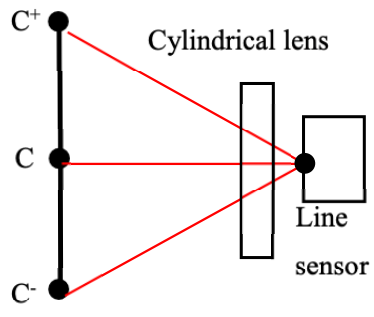

(b) Side view

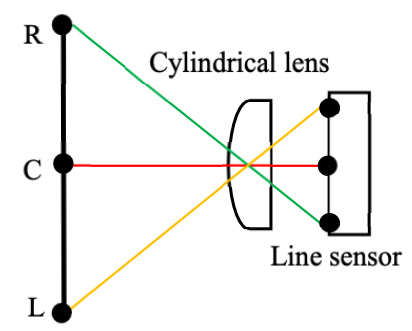

(c) Top view
Figure 2: Schematics of the One-Dimensional brightness distribution sensor.

distance from the sensor.

\subsection{Method of Finding the Difference between Normal} and Dangerous Gait Using Obrid-Sensor For distinguishing normal and dangerous gaits, the heights less than $10 \mathrm{~mm}$ must be extracted from the sensor data. We considered that combining the Obrid-Sensor, an infrared line laser, and an infrared transmitting filter would extract the horizontal brightness alone. The infrared line laser emits a horizontal line of laser light. Meanwhile, the combined sensor and infrared transmitting filter admit only the infrared light. To extract the horizontal brightness at the threshold, we set both the infrared line laser and combined sensor-filter at the threshold height. When the subject entered the detection area, the infrared light was reflected and input to the ObridSensor. This reflected light is the horizontal component of the light within the threshold height. The subject's position is then found by background subtraction. In this setting, the infrared reflected light was increased by increasing the distance from the laser; the peak brightness value was inversely proportional to distance.

To detect dangerous walking (i.e., small MinTC) by the laser and sensor setup, we considered two trajectory patterns, Over MinTC and Under MinTC, around MinTC timing (see Fig. 4). During Push, two peaks corresponded to Push by one foot and FF by the other foot. The Push peak gradually decreased as the foot was lifted from the floor. During Load, two peaks were again observed, correspond-

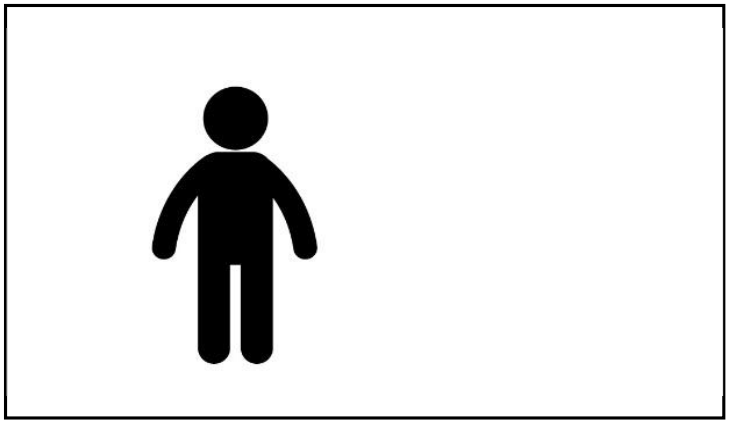

(a) Subject

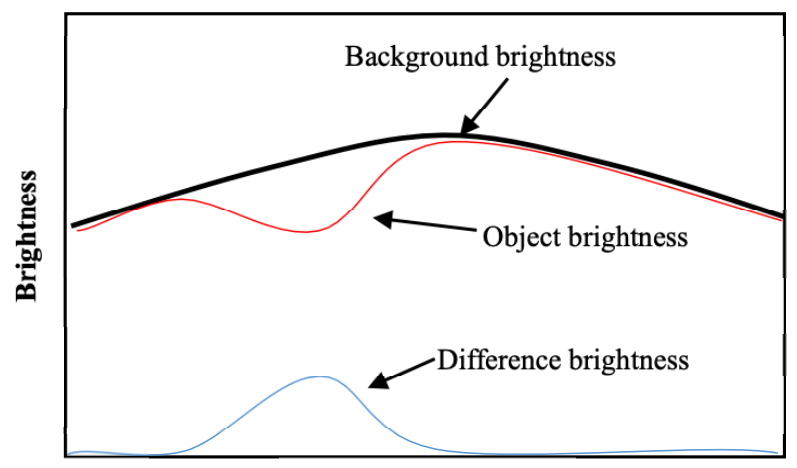

(b) Brightness distribution

Figure 3: Correspondence between the subject and brightness distribution.

ing to Load by one foot and FF by the other foot. Here, the Load peak gradually increased as the foot encountered the floor. During the swing phase, the brightness distribution exhibited a single peak. If the foot height was below the 10 $\mathrm{mm}$ threshold height, the brightness peak changed because infrared light was now reflected from the foot. Therefore, a dangerous gait was judged by a change in the brightness peak.

2.5 Judgement of the Dangerous Gait To judge normal and dangerous gaits, we focused on the size of the brightness distribution. The resulting brightnessdistribution size was obtained as the superimposed brightness distributions from the right and left feet. If the positions of the two feet were completely non-overlapping, the resulting brightness-distribution size was the summed brightness distributions of the right and left feet. Fig. 5 shows the theoretical time series of the brightness distribution size. Here, $h_{R}$ and $h_{L}$ are the summed brightness value when the right and left feet entered the detection area and became FF, respectively. The size of the brightnessdistribution area was acquired from the summed brightness distribution.

We first examine the time series of the brightnessdistribution size of the right foot during each walking phase. During Load, the size of the brightness distribution increases linearly as the foot descends to the floor, and reaches $h_{R}$ when the foot fully touches the floor. During FF, the size of the brightness distribution remains at $h_{R}$ because the foot does not move. During Push, the size of the brightness 


\section{Over threshold}

\section{Under threshold}
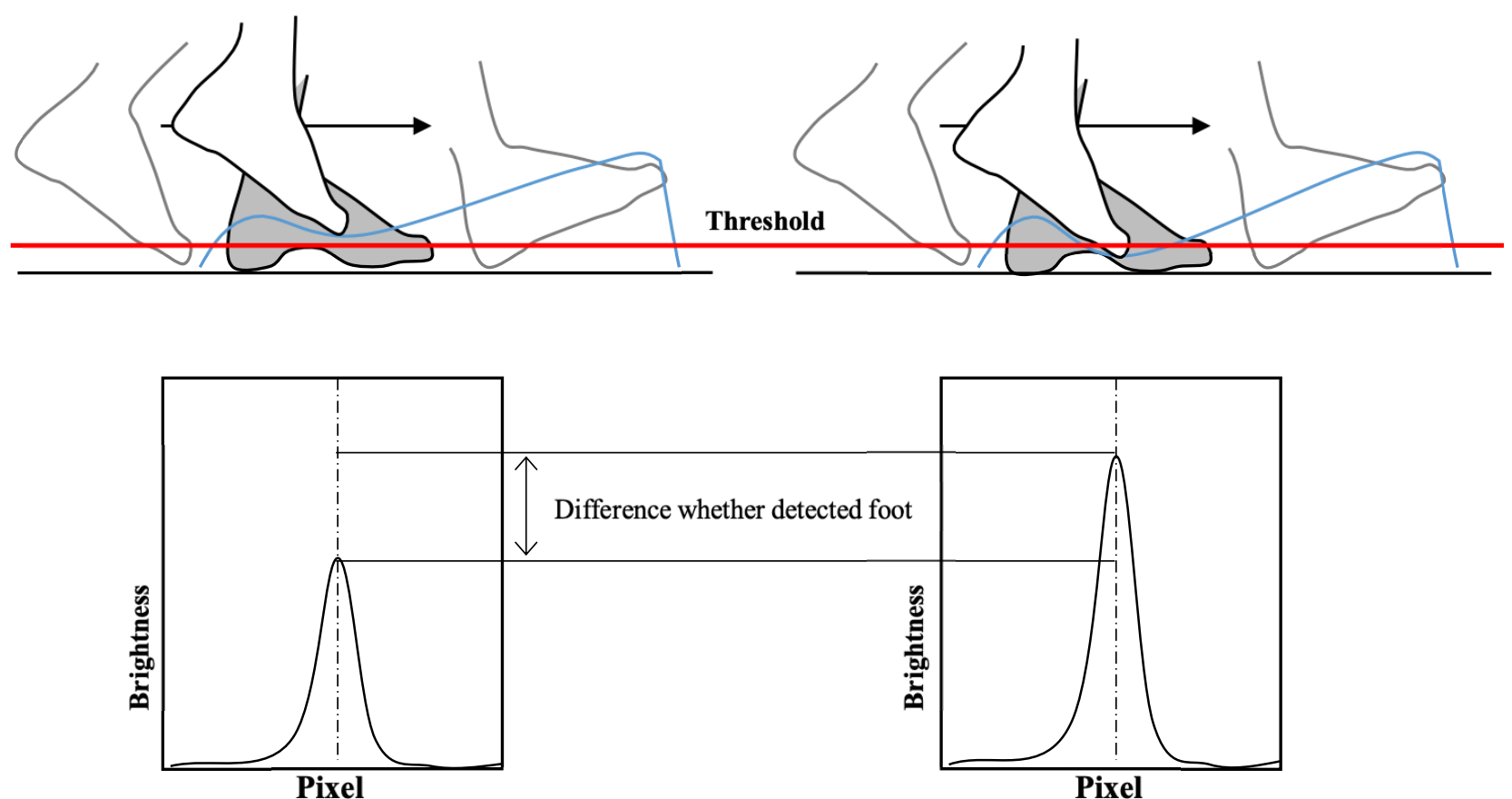

Figure 4: Brightness distributions during the swing phase of gait.

distribution decreases linearly to 0 finally because the foot gradually lifts from the floor. As time $t$ progresses, the size of the brightness distribution during each phase of the right foot $(f(t))$ is described by Eq. 5,6,7 and 8:

$$
\begin{aligned}
& f_{\text {Load }}(t)=a_{1} t \\
& f_{\mathrm{FF}}(t)=h_{R} \\
& f_{\mathrm{Push}}(t)=-a_{2} t \\
& f_{\mathrm{Swing}}(t)=0
\end{aligned}
$$

where $f_{\mathrm{Load}}(t), f_{\mathrm{FF}}(t), f_{\mathrm{Push}}(t)$ and $f_{\mathrm{Swing}}(t)$ denote the during Load, FF, Push and Swing, respectively, and $\mathrm{TS}_{R}, \mathrm{HS}_{R}$, $\mathrm{TO}_{R}$, and $\mathrm{HO}_{R}$ are the TS, HS, TO and $\mathrm{HO}$ of the right foot, respectively. $a_{1}$ and $a_{2}$ are the constants of proportionally during the Load and Push actions of the right foot, respectively given by Eq. 9 and 10:

$$
\begin{aligned}
& a_{1}=\frac{h_{R}}{t\left(\mathrm{TS}_{R}\right)-t\left(\mathrm{HS}_{R}\right)} \\
& a_{2}=\frac{h_{R}}{t\left(\mathrm{TO}_{R}\right)-t\left(\mathrm{HO}_{R}\right)}
\end{aligned}
$$

Second, we examine the time series of the brightnessdistribution size of the left foot. Similarly to the right foot, we define $b_{1}$ and $b_{2}$ as constants of proportionally during the Load and Push action of the left foot, respectively, and $\mathrm{TS}_{L}, \mathrm{HS}_{L}, \mathrm{TO}_{L}$, and $\mathrm{HO}_{L}$ as the TS, HS, TO and $\mathrm{HO}$ of the left foot, respectively. The size of brightness distribution during each phase from left foot $(g(t))$ is governed by Eq. 11, Eq. 12, Eq. 13, Eq. 14, Eq. 15 and Eq. 16:

$$
\begin{aligned}
& g_{\text {Load }}(t)=b_{1} t \\
& g_{\mathrm{FF}}(t)=h_{L} \\
& g_{\text {Push }}(t)=-b_{2} t \\
& g_{\text {Swing }}(t)=0 \\
& b_{1}=\frac{h_{L}}{t\left(\mathrm{TS}_{L}\right)-t\left(\mathrm{HS}_{L}\right)} \\
& b_{2}=\frac{h_{L}}{t\left(\mathrm{TO}_{L}\right)-t\left(\mathrm{HO}_{L}\right)}
\end{aligned}
$$

where $g_{\text {Load }}(t), g_{\mathrm{FF}}(t), g_{\mathrm{Push}}(t)$ and $g_{\mathrm{Swing}}(t)$ are denote $g(t)$ during Load, FF, Push and Swing, respectively.

Finally, the time series of the brightness-distribution size during walking is obtained by summing the brightness distributions of both feet as follows:

We now suggest a procedure for judging dangerous gaits from $A(t)$. When the Obrid-Sensor detects a dangerous gait as shown in 2.4, $A(t)$ increases. At this time, one foot is FF and the other foot is Load or Swing or Push. Accurately extracting the duration of the dangerous gait is difficult, because we cannot know the timing of the dangerous gait. For this reason, we consider the two peaks (the last peak $P_{1}$ and the next peak $P_{2}$ ) when both feet are in the stance phase. The duration of the dangerous gait is assumed to lie within 


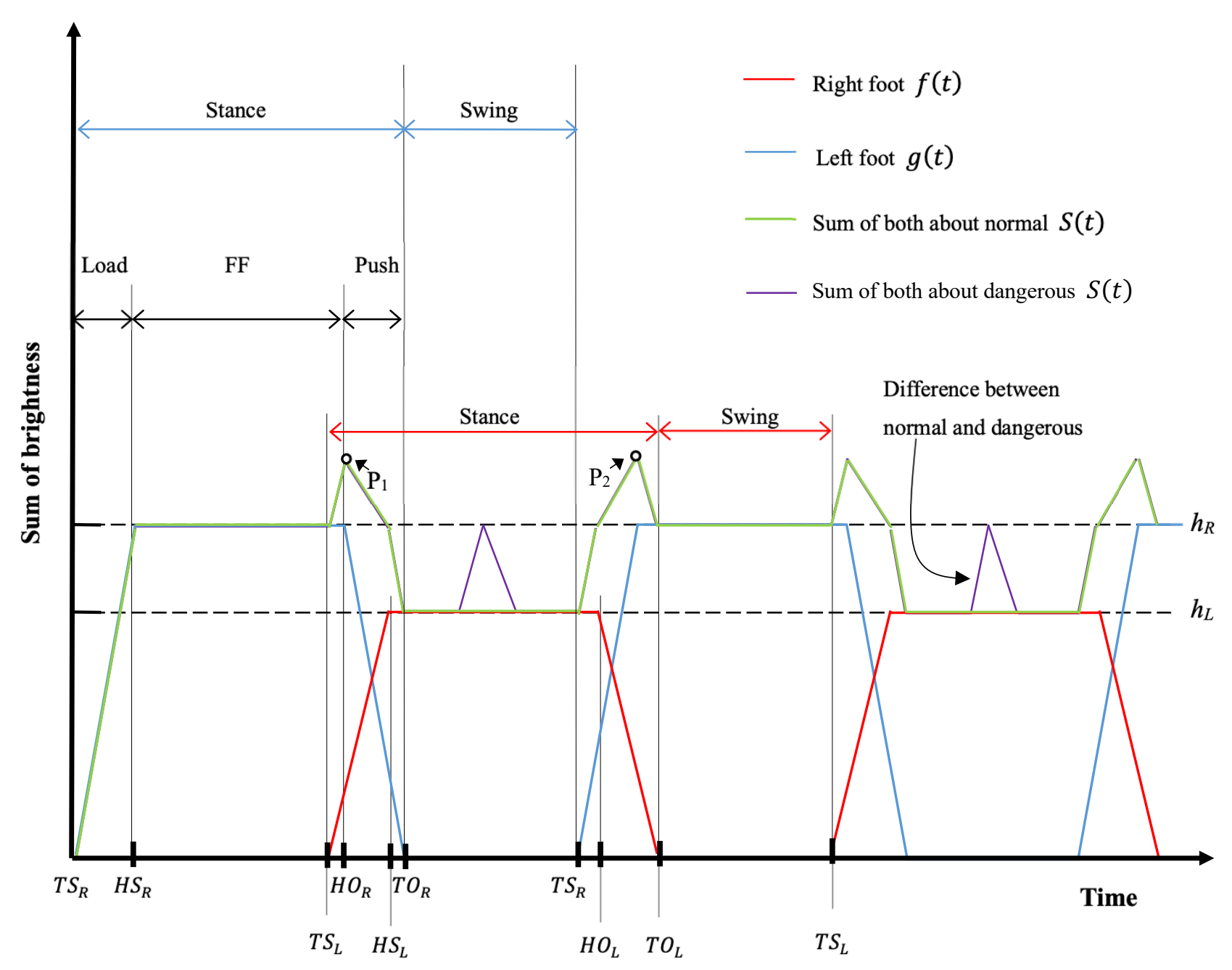

Figure 5: Time series of the summed of brightness distributions.

the time between these peaks. First, $\mathrm{n}$ data $\left\{A_{1}, A_{2} \ldots A_{n}\right\}$ are obtained in the duration between $P_{A}$ and $P_{B}$. The number of data $n$ depends on the walking speed and sampling period. Second, the average of $A\left(A_{M E A N}\right)$ is calculated for each gait. Finally, whether the $A_{M E A N}$ values differ between the normal and dangerous gaits is evaluated by a t-test.

\section{Experiment}

To confirm the increasing brightness when the foot descends below $10 \mathrm{~mm}$, we conducted a walking trial and obtained the time series data of the summed distributions by the proposed method during both normal and dangerous walking. We roughly estimated the swing phase and compared the brightness distributions of the normal and dangerous gaits, thus confirming the detection of the foot under 10 $\mathrm{mm}$. Finally, the significance difference between two gaits, determined by the methods in 2.4 and 2.5 , was verified in a t-test. In all experiments, we videoed the walking with a camera and confirmed whether the subjects followed instructions.

3.1 Experimental Environment The subjects were 6 healthy males in their twenties. Prior to enrolment, all subjects were informed of the purpose, contents, and bod- ily effects of the trial. The experiment was performed in an indoor lit environment, as shown in Fig. 6. The arrow in Fig. 6 indicates the traveling direction. We placed markers to guide the subject's gait. The markers were placed at equal intervals, and the sensor could detect feet when a foot was placed on markers 2, 3, and 4. Fig. 7 shows the Obrid-Sensor used in experiment. The cylindrical lens $(\mathrm{N}-\mathrm{SF} 1112.5 \times 25.0$, Edmund optics, USA) has a focal length of $12.5 \mathrm{~mm}$, a lens width of $12.5 \mathrm{~mm}$ and a radius of curvature of $9.81 \mathrm{~mm}$. Meanwhile, the line sensor (TSL1402, ams,Austria) of the Obrid-Sensor has the $256 \times 1$ pixel wide photo detectors, and a detection width of $18.3 \mathrm{~mm}$. Its data are processed by an AVR microcomputer (ARmega328P, Atmel, USA). The experiment used an infrared laser (B075TVTV1M, Summershine, China) with a was used in experiment with a center wavelength of $940 \mathrm{~nm}$ and a power of $300 \mathrm{~mW}$. Fig. 8 shows the height-detection setting. The interval between the infrared line laser and the sensor was experimentally adjusted to maximize the detection sensitivity in preliminary experiments. The visible light was removed by infrared transmit filters (IR80, Fujifilm, Japan) placed over the lenses of the Obrid-Sensor and the camera. All subjects wore socks during the experiment. 


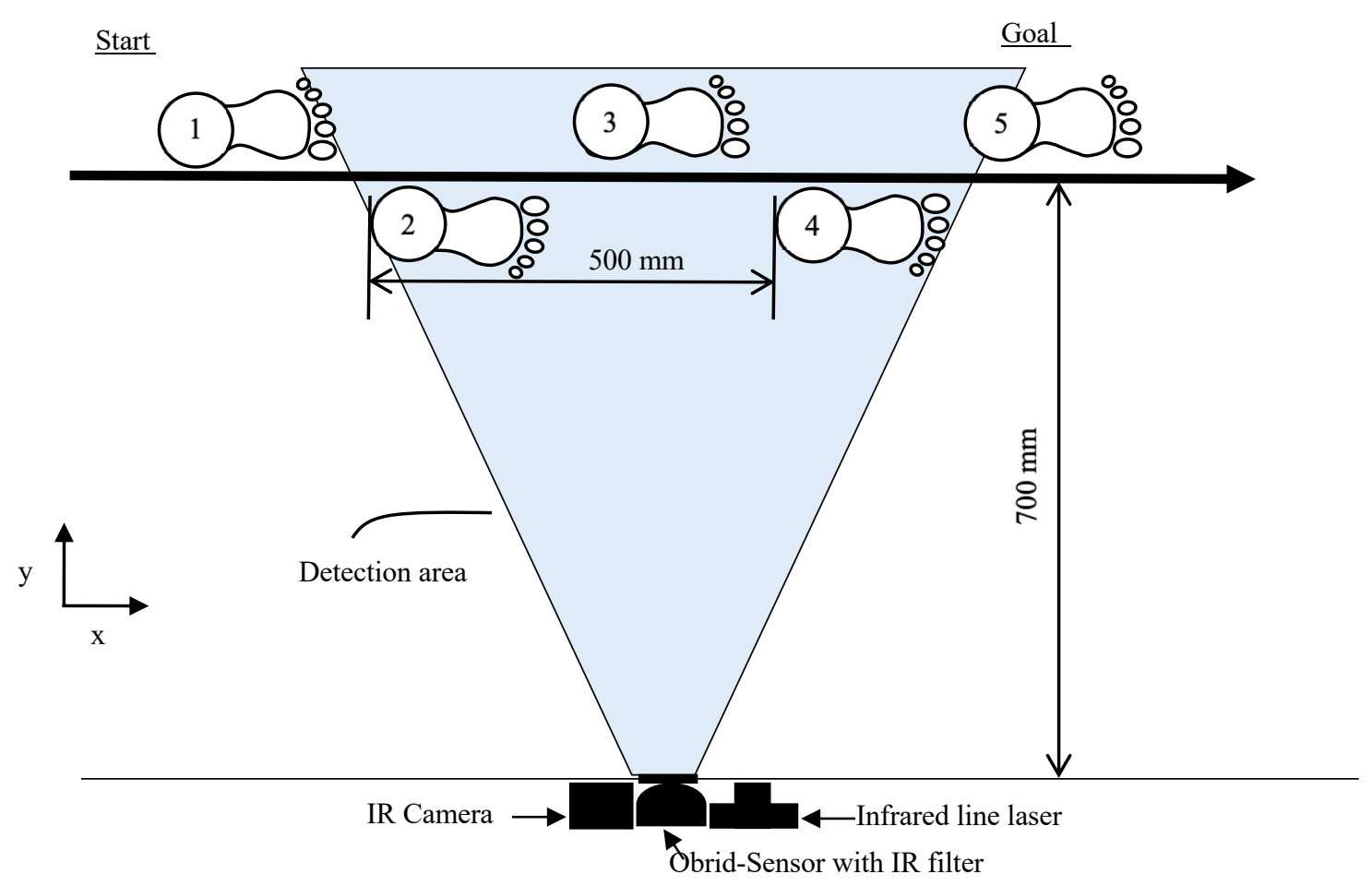

Figure 6: Schematic of the experimental environment.

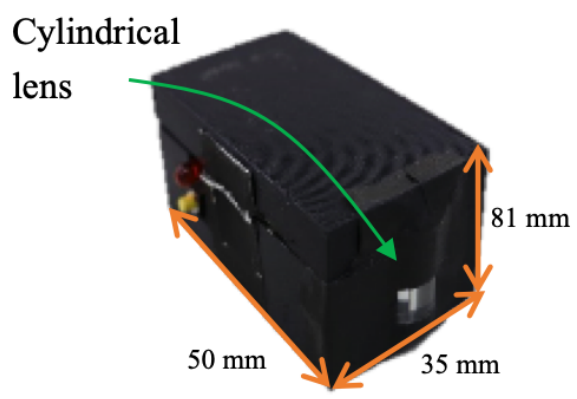

Figure 7: One-Dimensional brightness distribution Sensor.

3.2 Experimental Method The subject walked in front of the sensor. The start and goal positions of the trial are shown in Fig. 6. After receiving the start sign, the subject stepped onto the markers and the sensor obtained the brightness distribution until the subject reached the goal maker. In this trial, the subject was instructed to raise his foot above $10 \mathrm{~mm}$ as normal gait, and to walk at a preferably slow pace. This trial was performed five times. The subject then repeated the five trials with his foot raised below 10 $\mathrm{mm}$ as dangerous gait. In all walking trials, the walking speed was roughly constant, controlled by setting the same time on the stopwatch.

\section{Results}

Consist with Fig. 4, the brightness peak was higher when detecting a foot below $10 \mathrm{~mm}$ than when detecting a higher foot. Fig. 9 shows the brightness distributions during the swing phases of the normal and dangerous gaits.

The brightness values of the two feet were summed in each trial. As an example, Fig. 10 shows two time series

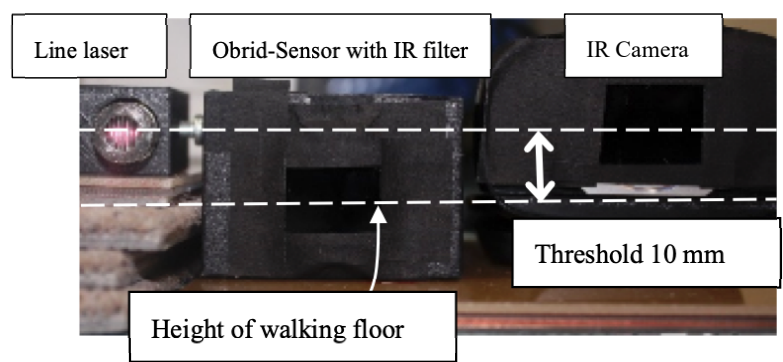

Figure 8: Settings of the sensor and laser height.

of the calculation result $(A(t))$, the normal and dangerous gaits. The y axis was normalized $A(t)$ for comparing the waveforms. $P_{1}$ and $P_{2}$ were obtained from $A(t)$, and $A_{M E A N}$ was calculated from the results of each trial. Fig. 11 shows the results of the t-test, the mean values, and standard deviation of $A_{M E A N}$. The $A_{M E A N}$ values of the normal and dangerous gaits were significantly different $(\mathrm{p}<0.05)$ in five subjects out of six subjects.

\section{Discussion}

Figure 9 confirms a large difference between the brightness distributions of the normal and dangerous gaits. The dangerous gait yielded two peaks in the brightness distribution, one at 155 pixels and the other at 215 pixels. The distributions of both gaits yielded a local maximum around 125 pixels, most likely caused by reflection light from the same point of the left foot. This brightness increase was seen in all results, implying that the proposed system can detect the right foot when it descends below the threshold height.

A local maximum (corresponding to $P_{1}$ ) in Fig. 10 appeared at approximately $2.8 \mathrm{~s}$ in the normal gait $\left(P_{1 A}\right)$ and 


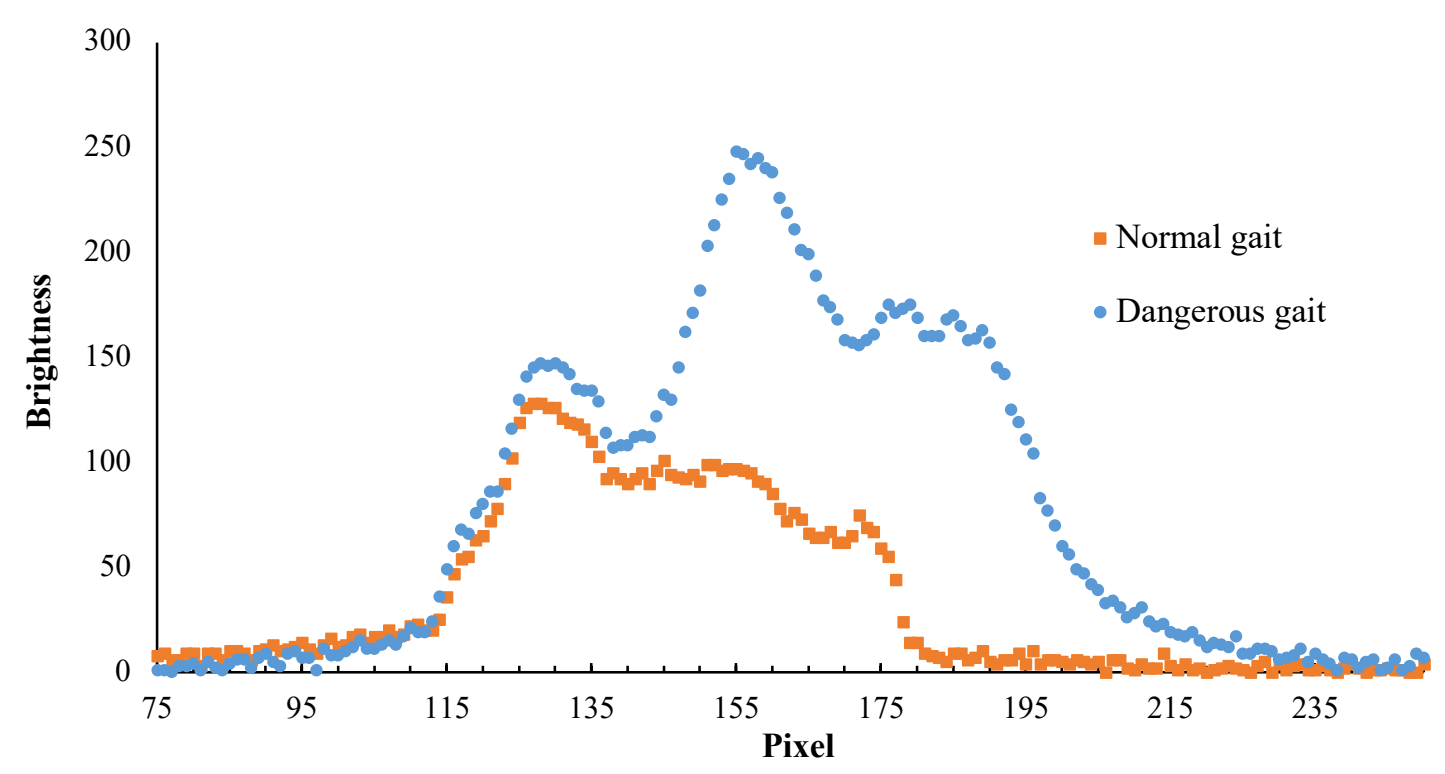

Figure 9: Brightness distributions, normal and dangerous gait.

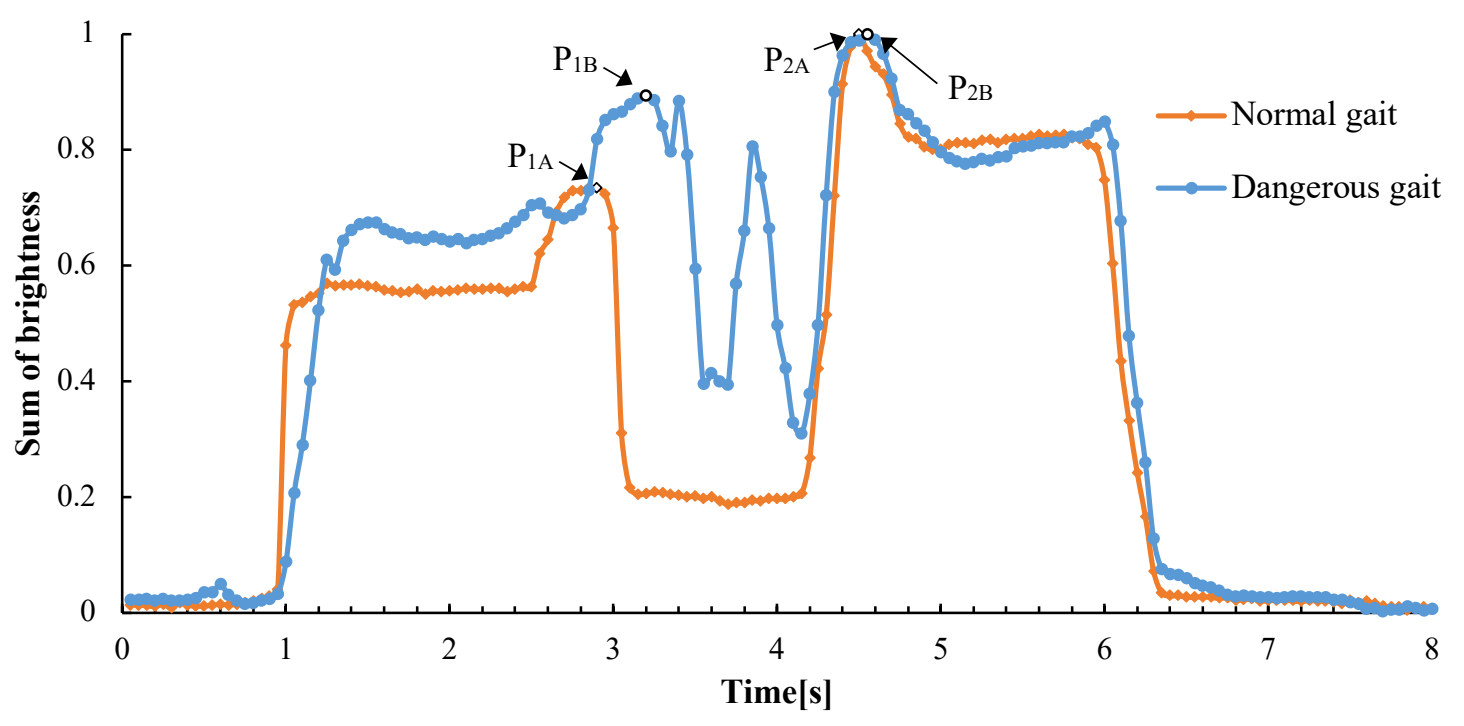

Figure 10: Time series of the summed brightness distributions of normal and dangerous gaits.

at $3.2 \mathrm{~s}$ in the dangerous gait $\left(P_{1 B}\right)$. At these times, the sensor system detected both feet. In the normal gait, the intensity distribution remained flat from $3.0 \mathrm{~s}$ to $4.2 \mathrm{~s}$, then rose to a local maximum at $4.5 \mathrm{~s}\left(P_{2 A}\right)$, again because both feet were detected. In the dangerous gait, the waveform repeatedly increased and decreased within the 3.2-4.2 s period. This behavior is attributable to the changing brightness reflected from a foot during swing phase. The final local maximum $\left(P_{2 B}\right)$ appeared when the foot opposing the one placed on the preceding marker was placed on the floor. Therefore, the large difference discussed in subsection 2.4 was confirmed by comparing the waveforms of the normal and dangerous gait.

The t-test confirmed a significant difference in the normal and dangerous gaits of five subjects out of six subjects (Fig. 11), verifying that the proposal method can usually distinguish between the two gait types. To resolve the failure of the proposed method on Subject 4, calculation could be refined to extract the swing phase. In future work, we should incorporate the position of the brightness peak into current method; we could then judge where the foot influences the brightness. The influences of the brightness from the room should also be distinguished from those of footwear.

Recently, laser range finders (LRFs) have become available for gait measurements. An LRF obtains the data per unit square area using a laser. However, the vertical detection error of these LRFs (HOKUYO, UAM-05LP-T301) is $30 \mathrm{~mm}$, larger than the reported range of mean MinTCs (10-24 mm). Therefore, the characteristic MinTC cannot be determined by LRFs, whereas the detection width of our line laser is comparable to the range of mean MinTCs. In 


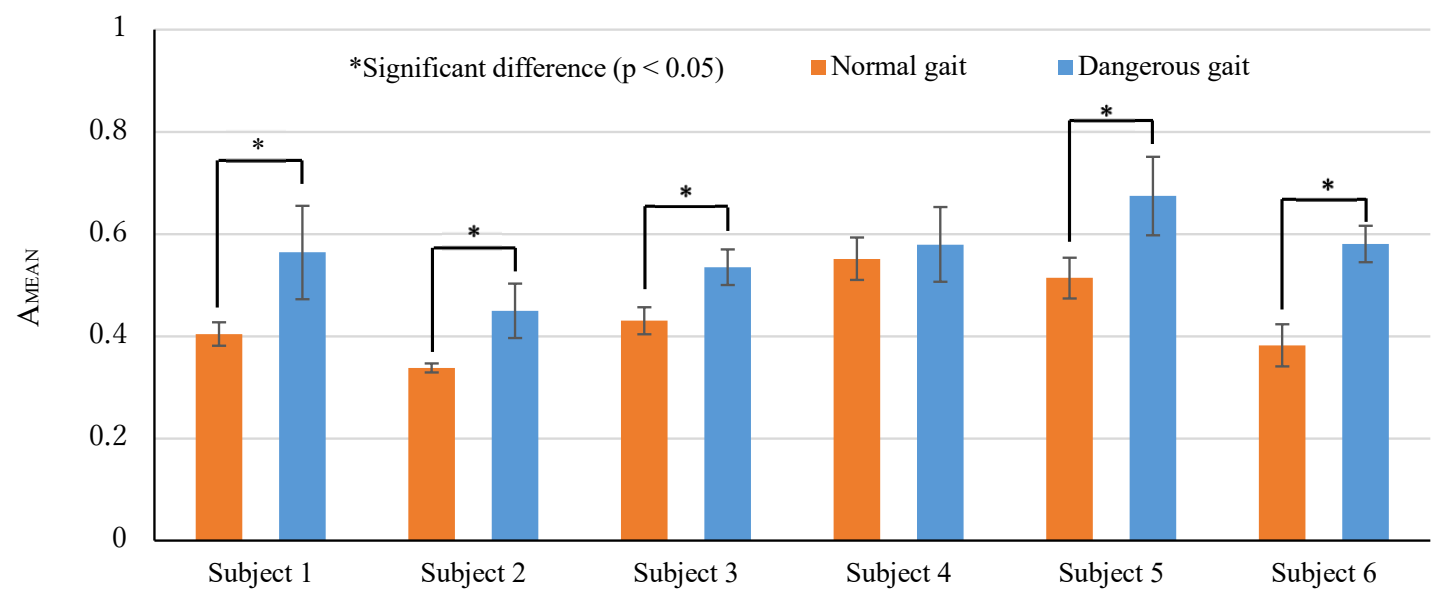

Figure 11: Time series of the summed brightness distributions of normal and dangerous gaits

future work, we must expand the effective range of our approach. The LRF-based gait measurement system of Ozawa [24]has an effective detection range of $5.0 \mathrm{~m}$, whereas the effective detection range of our method is limited by the line laser power. A large detection width is essential for actual environmental applications.

\section{Conclusions}

Despite its simplicity, our method reasonably distinguished between normal gaits with low fall risk and restricted gaits with high fall risk. Therefore, it can sufficiently detect dangerous gaits. Unlike current methods based on laser range finding, the spatial resolution of our method is sufficient for detecting the minimum toe clearance. In future work, we will expand the effective detection range, enabling use of the method in real environments.

\section{References}

[1] Suzuki T, Sugiura M, Furuna T, Nishikawa S, Yoshida H, Ishizaki T, Kim H, Yukawa H and Shibata H, "Association of physical performance and palls among the community elderly in Japan in a five year follow-up study", Japanese Journal of Geriatrics, Vol.36, No.7, pp.472-478, 1999. DOI: 10.3143 /geriatrics. 36.472

[2] World Health Organization, Fact sheets: Falls, http://www. who.int/news-room/fact-sheets/ detail/falls, access date: 2019.9.23

[3] Kanae Takahashi, Yoshimasa Nagao, Yuki Adachi, Takeshi Morimoto, Noriaki Ichikawa, Tadao Tsuboyama, Takashi Omori and Tosiya Sato, "Changes in the Dose of Benzodiazepines and Falls in Elderly Inpatients in an Acutecare Hospital", Japanese Journal of Pharmacoepidemiology, Vol.16, No.1, pp.11-20, 2011. DOI: 10.3820/jjpe.16.11

[4] R.S. Barrett, P.M. Mills and R.K. Begg, "A systematic review of the effect of ageing and falls history on minimum foot clearance characteristics during level walking",
Gait \& Posture, Vol.32, No.4, pp.429-435, 2010. DOI: 10.1016/j.gaitpost.2010.07.010

[5] S.W. Muir-Hunter and J.E. Wittwer, "Dual-task testing to predict falls in community-dwelling older adults: a systematic review", Physiotherapy, Vol.102, No.1, pp.29-40, 2016. DOI: 10.1016/j.physio.2015.04.011

[6] Yoshiyuki Kobayashi, Satoru Hashizume, Hiroaki Hobara, Emi Anzai, Kanako Nakajima, Koji Mishima, Kazuma Morizono, Aiichiro Sato and Masaaki Mochimaru, "Effect of safety boots with toe spring on foot clearance features during walking”, International Journal of Industrial Ergonomics, Vol.71, pp.32-36, 2019. DOI: 10.1016/j.ergon.2019.02.009

[7] Mifuka Ouchi, Nozomi Maeda, Kaori Sato and Hiroyuki Tokinaga, "The effect of Visual Information on ToeClearance when Stepping Over an Obstacle", Jpn Journal of Physical Therapy Science, Vol.27, No.2, pp.189-193, 2012. DOI: 10.1589/rika.27.189

[8] Tinetti ME, Speechley M and Ginter SF, "Risk factors for falls among elderly persons living in the community", $N$ Eng J Med, Vol.319, No.26, pp.1701-1707, 1988. DOI: 10.1056/NEJM198812293192604

[9] Siv Sadigh, Anne Reimers, Ragnar Andersson and Lucie Laflamme, "Falls and fall-related injuries among the elderly: a survey of residential-case facilities in a Swedish municipality", Journal of Community Health, Vol.29, No.2, pp.129140, 2004. DOI: 10.1023/b:johe.0000016717.22032.03

[10] Japanese Society of Physical Therapy, "Japanese Guidelines for the Physical Therapy", 2012.

[11] Naoya Katsurashita, Hiroaki Yamasaki, Takashi Kamiya, Naoyuki Chiba, Teruyoshi Endo and Makoto Ota, "The effect of walker exercise on light feedback device", Journal of Kochi Rehabilitation Institute, Vol.9, pp.23-27, 2008. DOI: 10.15028/kochireha.9.0_23

[12] Keiichi Hirasawa, Narimune Matsumura, Manabu Motegi, Tomohiro Yamada, Shinyo Muto, Naoyoshi Kanamaru, Kenichiro Shimokura, Masanobu Abe, Yoshiko Morita and Keiko Kasai, "Analyzing Rising Patterns of Patient to Prevent Bed-related Falls -First Report-", The Journal of Japan 
Society for Health Care Management, Vol.11, No.1, pp.3135, 2010.

[13] Daniel T.H. Lai, Simon B. Taylor and Rezaul K. Begg, "Prediction of foot clearance parameters as a precursor to forecasting the risk of tripping and falling", Human Movement Science, Vol.31, No.2, pp.271-283, 2012. DOI: 10.1016/j.humov.2010.07.009

[14] Yoshiyuki Kobayashi, Kei Aoki, Eijiro Shibusawa and Masaaki Mochimaru, "Proposal of the minimum toe clearance estimation method using floor reaction force", Journal of the Society of Biomechanisms, Vol.37, No.4, pp.233-242, 2013. DOI: https://doi.org/10.3951/sobim.37.233

[15] Yasuaki OHTAKI, Koichi SAGAWA and Hikaru INOOKA, "A Method for the Long-Term Gait Assessment Utilizing Accelerometers and Gyroscopes", Transactions of the Japan Society of Mechanical Engineers, Vol.67, No.655, pp.782788, 2001. DOI: 10.1299/kikaic.67.782

[16] Yoshihiro Ehara and Sumiko Yamamoto, "rinshohokoukeisokunyumon(Introduction to clinical gait measurement)", Ishiyaku publishers, 2008(in Japanese).

[17] Benoit Mariani, Hossein Rouhani, Xavier Crevoisier and Kamiar Aminian, "Quantitative estimation of foot-flat and stance phase of gait using foot-worn inertial sensors", Gait \& Posture, Vol.37, No.2, pp.229-234, 2013. DOI: 10.1016/j.gaitpost.2012.07.012

[18] Hanatsu Nagano, Rezaul K. Begg, William A. Sparrow and Simon Taylor, "Ageing and limb dominance effects on footground clearance during treadmill and overground walking", Clinical Biomechanics, Vol.26, No.9, pp.962-968, 2011. DIO: $10.1016 /$ j.clinbiomech.2011.05.013

[19] Mufadal A. Moosabhoy and Steven A. Gard, "Methodology for determining the sensitivity of swing leg toe clearance and leg length to swing leg joint angles during gait", Gait \& Posture, Vol.24, No.4, pp.493-501, 2006. DOI: 10.1016/j.gaitpost.2005.12.004

[20] David A Winter, "Foot Trajectory in Human Gait: A precise and Multifactorial Motor Control Task", Physical Therapy, Vol.72, No.1, pp.45-53, 1992. DOI: 10.1093/ptj/72.1.45

[21] Mills PM, Barrett RS and Morrison S, "Toe clearance variability during walking in young and elderly men", Gait \& Posture, Vol.28, No.1, pp.101-107, 2008. DOI: 10.1016/j.gaitpost.2007.10.006

[22] Masayuki Soma, "Toe clearance and toe gripping strength in walking -a study for fall prevention-", Japanese Journal of Health Promotion and Physical Therapy, Vol.6, No.1, pp.17, 2016. DOI: 10.9759/hppt.6.1

[23] Shota Nakashima, Yuhki Kitazono, Kanya Tanaka and Yuji Wakasa, "A Proposal of Method to Estimate Person's Upright and Falling Using the On-Dimensional Brightness Distribution Sensor", The Japanese Journal of the Institute of Industrial Applications Engineers, Vol.1, No.1, pp.20-26, 2013. DOI: $10.12792 /$ jjiiae.001.01.003

[24] Mayumi Ozawa, Ayanori Yorozu, Tetsuya Matsumura and Masaki Takahashi, "Gait mesurement system using laser range finder", Transactions of the Japan Society of Mechanical Engineers, Vol.79, No.801, pp.1550-1560, 2013. DOI: 10.1299/kikaic.79.1550

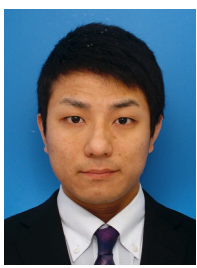

Daiki Hamada (Member) is graduated student of Electrical Engineering in National Institute of Technology, Ube College at 2018. He received a Associate degree in Electrical Engineering from National Institute of Technology. His current research interests include intelligent sensing and robotics. He is a member of the IIAE.

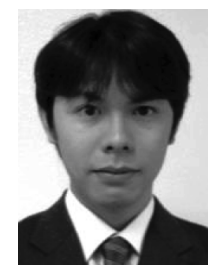

Shota Nakashima(Member) received the $\mathrm{Ph} . \mathrm{D}$. degree in electrical engineering from the Kyushu Institute of Technology, in 2010. From 2010 to 2012, he was an Assistant Professor with the Ube National College of Technology. $\mathrm{He}$ is currently a Senior Assistant Professor with Yamaguchi University. His current research interests include image processing, intelligent sensing, and artificial life. He is a member of the SCIE, IEEJ, and IIAE.

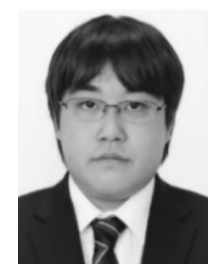

Tomohiro Morio(Member) received a B.E and M.E degree from Yamaguchi University, Yamaguchi, Japan, in 2017 and 2019. His current research interests include measurement, image processing, intelligent sensing and robotics.

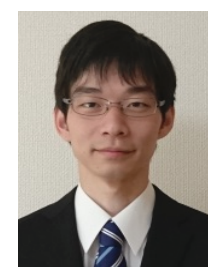

Tatsuya Sugimura (Member) received the B.E. degree in electrical system engineering from Yamaguchi University, Japan, in 2017, and the M.E. degree in the Graduate School of Science and Technology for Innovation from Yamaguchi University, Japan, in 2019. His research interests include intelligent sensing.

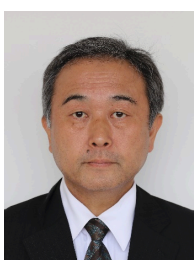

Kazuo Haruyama (Member) received the $\mathrm{Ph}$. D. degree in Graduate School of Science and Engineering, Yamaguchi University, in 2007. He is currently a professor with National Institute of Technology, Ube College. His current research interests include intelligent sensing for welfare. $\mathrm{He}$ is a member of the IEEJ and IIAE. 